\title{
Paeoniflorin Promotes Apoptosis of Fibroblast-Like Synoviocytes by Upregulating IncRNA MALAT1 to Inhibit Activation of the Wnt1/ $\beta$-Catenin Pathway in Collagen-Induced Arthritis Rats
}

\section{Fan Yang ( $\sim$ junkifan626@163.com )}

Nanjing Jinling Hospital: East Region Military Command General Hospital https://orcid.org/00000002-8679-4803

\section{Dongyi Wang}

Nanjing Jinling Hospital: East Region Military Command General Hospital Junyi Shen

Nanjing Jinling Hospital: East Region Military Command General Hospital Jun Han

Jiangsu Provincial Hospital of Integrated Chinese and Western Medicine: Jiangsu Province Academy of Traditional Chinese Medicine

\section{Kailun Zhou}

Nanjing Jinling Hospital: East Region Military Command General Hospital

\section{Zhiming Zhao}

Nanjing Jinling Hospital: East Region Military Command General Hospital

Hui Cai

Nanjing Jinling Hospital: East Region Military Command General Hospital

\section{Wei Shang}

Nanjing Jinling Hospital: East Region Military Command General Hospital https://orcid.org/00000002-0086-0436

\section{Research Article}

Keywords: Paeoniflorin, Rheumatoid arthritis, Fibroblast-like synoviocytes, LncRNA MALAT1, apoptosis, Wnt1/ $\beta$-catenin pathway

Posted Date: February 9th, 2022

DOI: https://doi.org/10.21203/rs.3.rs-1320331/v1

License: (c) (i) This work is licensed under a Creative Commons Attribution 4.0 International License. Read Full License 
Page $2 / 22$ 


\section{Abstract}

This study explored the effect of paeoniflorin(PAE) on apoptosis of fibroblast-like synoviocytes(FLS) mediated by the Wnt1/ $\beta$-catenin pathway in collagen-induced arthritis(CIA) rats based on the expression of IncRNA MALAT1. Sprague-dawley(SD) rats were randomly divided into control group, CIA model group, PAE low-dose group, PAE medium-dose group and PAE high-dose group. Quantitative polymerase chain reaction (qPCR) was then performed to determine the expression of MALAT1 in rat FLS. Next, the apoptosis rate of FLS was detected by terminal-deoxynucleotidyl transferase-mediated nick end labeling(TUNEL), whereas cell morphology was observed by electron microscopy. Enzyme-linked immunosorbent assay (ELISA) was used to determine the levels of TNF $-a$, IL-6, and IL-2 cytokines. The mRNA expressions of Wnt1, $\beta$-catenin, Caspase-3, Caspase-9, Bcl-2 and Bax were determined by qPCR, whereas the protein expressions of Wnt1, $\beta$-catenin, Caspase-3, Caspase-9, Bcl-2 and Bax were measured by western blot analysis. PAE alleviated arthritis in CIA rats in a dose-dependent manner. In CIA rats, PAE reduced arthritis scores and decreased joint histopathological changes. The expression level of MALAT1 was lower in the CIA group compared with the control group, but the expression was reversed after PAE treatment. The expression levels of Wnt1 and $\beta$-catenin in the CIA group were increased compared with the control group, and the levels of cytokine TNF $-a$, IL-6, and IL-2 were raised compared to the control group. However, treatment with PAE decreased the levels of all these cytokines. Besides, PAE could improve the cell apoptosis rate, and increase the expression levels of Caspase-3, Caspase-9 and Bax, reducing the expression level of Bcl-2. PAE may promote the apoptosis of RA-FLS by up-regulating MALAT1 to inhibit activation of the Wnt1/ $\beta$-catenin pathway, thereby alleviating arthritis in CIA rats.

\section{Introduction}

Rheumatoid arthritis (RA) is a systemic autoimmune disease characterized by joint damage, stiffness, and swelling [1, 2]. Fibroblast-like synoviocytes(FLSs) exhibit an epigenetic aggressive phenotype of RA, which transforms the synovium into proliferative infiltrating tissue, and ultimately leads to bone erosion and cartilage destruction[3]. With regard to the clinical manifestations, RA patients eventually develop progressive disability and complications, which seriously affect their quality of life [4]. To date, the conventional synthetic disease-modifying antirheumatic drugs (csDMARD), which mainly relieve the local symptoms, are still the mainstream RA treatment method. In recent decades, several biological agents, including tumor necrosis factor inhibitors (TNFi) and non-TNFi drugs, and targeted synthetic drugs such as Janus kinase (JAK) inhibitors have been gradually developed. Studies have shown that they produce anti-inflammatory effects by inhibiting cytokines associated with synovial inflammation [5]. However, in clinical treatment, these biological agents and targeted synthetic drugs are often not considered as firstline drugs due to inadequate response or intolerance to drugs and their very high cost, Which results in a risk of poor prognosis[6, 7]. Therefore, this calls for development of a safe, effective, and more economical way to suppress the joint structural damage in RA treatment.

Recent studies have shown that long non-coding RNA metastasis-associated lung adenocarcinoma transcript 1(IncRNA MALAT1) can inhibit the transcription and expression of FLS-related genes, thereby 
participating in the apoptosis and inflammatory reactions of FLS [8]. LncRNA MALAT1, with a length of about 8 KB in the human body, located on chromosome 11Q13, and widely expressed in normal tissues, is one of the research hotpots [9]. Notably, FLS is the main effector cell of RA leading to joint destruction, and has a unique invasive behavior in RA. Given its limited in situ proliferation ability and resistance to endoplasmic reticulum (ER) stress and migration of mesenchymal stem cells, FLS can resist apoptosis and activate high invasion and migration ability to surrounding bone and cartilage[3]. This suggests that targeted IncRNA MALAT1 therapy may inhibit the pathological changes of FLS, thereby achieving the effect of remission or even RA treatment.

Paeoniflorin (PAE) is a water-soluble monoterpene glycoside, which has been used to treat immune diseases for more than a thousand years in China[10]. Modern pharmacological studies have confirmed that PAE has anti-inflammatory, antioxidative, autophagy modulation, and neuroprotection abilities [1113]. One study reported that PAE plays a critical role in the anti-inflammatory and immunomotor mechanisms by reducing abnormal signal transduction and regulating the function of immune cells[10]. However, although the pharmacological role of PAE in RA treatment has been gradually confirmed by various studies, there is no report on whether PAE participates in MALAT1-targeted RA treatment.

In our previous in vitro study (currently under review for publication), we found that IncRNA MALAT1 expression was down-regulated in the RA-FLS group compared to the control group, whereas PAE upregulated the expression of IncRNA MALAT1, and promoted the apoptosis in the RA-FLS group. Based on these findings, we hypothesized that PAE may target MALAT1 to promote apoptosis of RA-FLS. Consequently, this study conducted in vivo experiments to evaluate the effect of PAE on articular inflammation in CIA rat models, and explored the effects of PAE on IncRNA MALAT1 expression, cell apoptosis, and potential signaling pathways in vivo, with the overarching goal of providing new evidence to support PAE treatment of RA.

\section{Materials And Methods}

\section{Reagents}

PAE(>98\%pure) was supplied by Shanghai Yuanye Biotechnology Co., Ltd. (Shanghai, China). DMEM, fetal bovine serum, penicillin and streptomycin were purchased from Hyclone Biotechnology Co., Ltd. (Logan, UT, USA). Phosphate-buffered saline(PBS) was purchased from Nanjing Shengxing Biotechnology Co., Ltd. (Nanjing, Jiangsu, China). Trizol, PrimeScript ${ }^{\mathrm{TM}} \mathrm{RT}$ reagent Kit with gDNA Eraser were purchased from Takara Biotechnology Co., Ltd. (Tokyo, Japan). NanoDrop 2000 spectrophotometer was purchased from Thermo Fisher Scientific(Waltham, MA, USA). Fluorescence quantitative PCR was purchased from Applied Biosystems. Wnt1 primary antibody was obtained from Abcam(Cambridge, UK), Bax, Bcl2 and $\beta$-catenin primary antibodies were purchased from Protein Tech(Chicago, IL, USA), caspase3 and caspase9 primary antibodies were obtained from Biorbyt(Cambridge, UK). Bicinchoninic acid(BCA) protein assay kit was purchased from Beyotine. ECL luminous fluid was purchased from Tanon Biotechnology Co., Ltd. (Shanghai, China). IL-2 ELISA kit, IL-6 ELISA kit, TNF-a ELISA kit were 
purchased from Cusabio Technology(Beijing, China). Tanon ECL was used for chemiluminescence, and pictures were photoed by Tanon5200 chemiluminescence imager. Bovine type II collagen was purchased from Beijing biolead Technology Development Co., Ltd. (Beijing, China). Microscope was purchased from Nikon Corporation(Tokyo, Japan).

\section{Establishment of CIA rats model}

8-week-old female sprague-dawley(SD) rats were purchased from the experiment animal management department of Shanghai institute of family planning science(Shanghai, China). The protocol was approved by the Ethics Committee of Jinling Hospital.

Bovine type II collagen emulsifier was prepared to induce arthritis. In a sterile environment, $50 \mathrm{mg}$ bovine type II collagen was completely dissolved into acetic acid solution at a concentration of $2 \mathrm{mg} / \mathrm{mL}$, and then placed overnight in a refrigerator at $4^{\circ} \mathrm{C}$. The same volume of complete Freund's adjuvant and bovine type II collagen acetic acid solution were fully dissolved to prepare an emulsion with a concentration of $1 \mathrm{mg} / \mathrm{mL}$. The rats were divided into ctrl group and CIA group, and the CIA group was injected with $0.2 \mathrm{~mL}$ emulsion at tail root on day 0 and 7, respectively. Ctrl group rats were injected with the same amount of normal saline. From the 10th day of modeling, arthritis score was performed twice a week according to erythema, swelling and stiffness of ankle joint. After successful modeling of CIA, the rats were divided into 5 groups: RA model control group, CIA group and 3 doses of PAE treatment group $(50 \mathrm{mg} / \mathrm{kg}, 100 \mathrm{mg} / \mathrm{kg}, 150 \mathrm{mg} / \mathrm{kg}$ )[14]. The 3 doses of PAE treatment group were respectively represented as PAE-low, PAE-middle, PAE-high.

\section{Arthritis assessment indicators}

The rat arthritis index is based on a cumulative score that is the degree of swelling in the joints of the extremities and is expressed on a scale of $0-4$, with 4 being the most inflammatory. Therefore, the maximum cumulative score for each rat is 16 . The detailed scoring criteria are as follows: $0=$ normal or no inflammation; $1=$ slight redness or swelling of the little toe joint; $2=$ swelling and redness of the little toe joints and toes; $3=$ swelling and redness below the ankle; $4=$ severe swelling and redness of the ankle joint, and even severe joint deformation[15]. The joint inflammation index was recorded every 5 days from the day of successful modeling.

The volume below the ankle was measured with a hind limb volumeter and paw swelling degree was obtained by the following formula: $V(\%)=\left(V_{\text {afte }}{ }^{r-V_{\text {before }}}\right) / V_{\text {before }} \times 100 \%$. With the same frequency as the arthritis index, paw swelling(\%) was recorded every 5 days.

\section{Histopathological analysis}

The fresh synovial tissues from the rats model were fixed in $4 \%$ paraformaldehyde for $24 \mathrm{~h}$ and dehydrated by gradient ethanol in turn. The wax-soaked tissues were embedded in the embedding machine, and the finished wax blocks were placed on the paraffin slicer for sectioning with a thickness of $4 \mu \mathrm{m}$. Then the nucleus was stained with hematoxylin, the cytoplasm was stained with eosin, and the images were collected and analyzed under a microscope. Scores from 0 to 4 indicate the severity of the 
lesion in turn, as assessed by the pathological appearance of arthritic lesions. Refer to the following criteria: $0=$ normal, $1=$ minimal inflammatory infiltration with pannus formation, $2=$ mild infiltration with superficial cartilage thinning, $3=$ moderate aggregates with lymphoid infiltration, cartilage zone damage and significant bone resorption, 4 = significant infiltration with edema, subchondral erosion and severe bone erosion[16].

Quantitative polymerase chain reaction (qPCR)

The purity and concentration of RNA were determined by NanoDrop 2000 spectrophotometer. According to the instructions of PrimeScript RT Reagent Kit with gDNA Eraser reverse Transcription Kit, PCR reaction conditions were $50{ }^{\circ} \mathrm{C}$ for $15 \mathrm{~min}$ and $85^{\circ} \mathrm{C}$ for $5 \mathrm{~min}$. PCR reaction procedure: denaturation at $95^{\circ} \mathrm{C}$ for $10 \mathrm{~min}$; $\left(95^{\circ} \mathrm{C}\right.$ denaturation for $15 \mathrm{~s}, 60^{\circ} \mathrm{C}$ annealing for $\left.1 \mathrm{~min}\right) \times 40$ cycles. With Glyceraldehyde-3phosphate dehydrogenase(GAPDH) as an internal reference, real-time PCR was performed by fluorescence quantitative PCR, and the results were calculated according to the $2^{-\Delta \Delta C t}$ formula. The primers of IncRNA MALAT1, Bax, Bcl2, caspase3, caspase9, and GAPDH were designed and synthesized by Enhance Biotechnology Co., Ltd. (Nanjing, China)(Table 1)

Table 1

Primer sequences for q-PCR

\begin{tabular}{|c|c|c|}
\hline & Forward & Reverse \\
\hline MALAT1 & GGGGGAATGGGGGCAAAATA & ACTGTAAACCTGGCGAGCTC \\
\hline Wnt1 & CAACATCGATTTCGGTCGCC & САCTCTTGGCGCATCTCAGA \\
\hline$\beta$-catenin & AAGGTGCTGTCTGTCTGCTC & CTAGAGTCCCAAGGAGGCCT \\
\hline Bax & GAACCATCATGGGCTGGACA & GTGAGTGAGGCAGTGAGGAC \\
\hline $\mathrm{Bcl} 2$ & СTTCTCTCGTCGCTACCGTC & СААТССТСССССАGTTСАСС \\
\hline caspase3 & ACTACTGCCGGAGTCTGACT & TAACCGGGTGCGGTAGAGTA \\
\hline caspase9 & TGGTTCTGGCAGAGCTCATG & AGCTTCTCACAGTCCACGTG \\
\hline GAPDH & TGATGGGTGTGAACCACGAG & AGTGATGGCATGGACTGTGG \\
\hline
\end{tabular}

FLSs were lysed using Radio Immunoprecipitation Assay(RIPA) lysis buffer on ice for 30min and centrifuged at $12,000 \mathrm{rpm}$ for $5 \mathrm{~min}$ at $4^{\circ} \mathrm{C}$ to obtain the supernatant. According to the instructions of the BCA kit to determine the protein content. The protein samples were separated by sodium dodecyl sulfate polyacrylamide gel electrophoresis(SDS-PAGE) and transferred to the PVDF membrane by electrophoresis. After soaking the membrane from bottom to top with TBS, the membrane was blocked with solution ( $5 \%$ defatted milk powder TBST solution) at room temperature for $1 \mathrm{~h}$ and incubated with the following primary antibodies overnight at $4^{\circ} \mathrm{C}$ :anti-Bax(1:2000), anti-Bcl2(1:1000), anti- 
caspase3(1:500), anti-caspase9(1:1000), anti-Wnt1(1:1000), anti- $\beta$-catenin(1:1000). Secondary antibodies that goat anti-rabbit IgG and goat anti-mouse IgG labeled by HRP were incubated with the membrane at $37^{\circ} \mathrm{C}$ for $2 \mathrm{~h}$.

\section{Isolation and culture of rat fibroblast-like synoviocytes}

Experiment after euthanasia by cervical dislocation, surgically remove tissue samples from the right ankle joint were taken and rinsed twice in PBS buffer containing penicillin-streptomycin to remove residual blood. The tissue was cut into the smallest possible blocks and transferred to a centrifugal tube. $0.1 \%$ collagenase IV was added to obtain FLS cells, then placed FLSs in a thermostatic shaker at $37^{\circ} \mathrm{C}$, shaking digestion at $150 \mathrm{rpm}$ for $2 \mathrm{~h}$. Filter with $100 \mu \mathrm{m}$ cell sieve, centrifugation at $1500 \mathrm{rpm}$ for $5 \mathrm{~min}$, the cell density was adjusted to $1 \times 10^{5}$ cells and inoculated into Petri dishes. The cells were cultured in a DMEM medium containing $10 \%$ fetal bovine serum, $100 \mathrm{U} / \mathrm{mL}$ penicillin, and $100 \mu \mathrm{g} / \mathrm{mL}$ streptomycin in a cell incubator containing $5 \% \mathrm{CO}_{2}$ at $37^{\circ} \mathrm{C}$. When the cells grew to $80-90 \%$, they were passed $1: 3$ and cultured in Petri dishes for follow-up experiments.

\section{Elisa}

The RA-FLS supernatant was obtained and tested the expression levels of IL-2, IL-6, TNF-a according to the corresponding enzyme-linked immunosorbent assay(ELISA) kit instructions. The diluent was added to a 96-well plate, incubated for $2 \mathrm{~h}$ at $37^{\circ} \mathrm{C}, 100 \mu \mathrm{L}$ biotin-labeled antibody was added to each well, then added $100 \mu \mathrm{L}$ HRP-labeled anti-biotin-labeled protein was to each well and cultured for $1 \mathrm{~h}$. After washing, $90 \mu \mathrm{L}$ TMB reaction substrate was added into each well, finally, $50 \mu \mathrm{L}$ termination buffer was mixed with them in each well after dark incubation. The absorbance was measured at $450 \mathrm{~nm}$ on the microplate reader.

\section{Tunel}

Apoptosis was detected by TUNEL assay. Following the instructions of the TUNEL assay kit, the fresh tissue was fixed in $4 \%$ paraformaldehyde for more than $24 \mathrm{~h}$. After dehydration, the working solution of protease $\mathrm{K}$ was dropped to cover the tissue (the storage solution of protease $\mathrm{K}$ was diluted with PBS 1:9), and the tissue was incubated for $30 \mathrm{~min}$ at $37^{\circ} \mathrm{C}$. Mixed with $50 \mu \mathrm{L} \mathrm{TdT}+450 \mu \mathrm{L}$ luciferase labeled dUTP solution, and incubate in a wet box at $37^{\circ} \mathrm{C}$ for 2 hours. After the slides were dried, added $50 \mu \mathrm{POD}$ to the specimens, and incubated for $30 \mathrm{~min}$ in a wet box at $37^{\circ} \mathrm{C}$. Fresh DAB chromogenic solution was dropped, and the positive results indicated that the nuclei were brownish yellow, and the sections were washed with running water to stop chromogenic. After the photo was taken, redye with hematoxylin or methyl green and rinse immediately with running water after a few seconds. Gradient alcohol dehydration, xylene transparent, neutral gum sealing sheet. Apoptotic cells were observed by a light microscope.

\section{Statistical analysis}


Graphpad Prsim 5.0(Graphpad Software, USA) was used for statistical analysis. The measurement data were represented as mean $\pm S D$, the difference between the two groups was evaluated by T-test, and the measurement data between multiple groups were compared by one-way ANOVA, $P<0.05$ was statistically significant.

\section{Results}

\section{LncRNA MALAT1 was low expression in fibroblast-like synoviocytes of CIA rats}

Fibroblast-like synoviocytes(FLS) were isolated and cultured from synovial tissues of rats, the FLS were divided into CIA and ctrl groups. The mRNA expression of MALAT1 in rat model FLS was detected by qPCR, and it was found that compared with the ctrl group, the expression level of IncRNA MALAT1 was significantly decreased in the CIA group(Fig. 1, P<0.01).

\section{PAE could up-regulate MALAT1 and promote apoptosis of FLS in CIA rats}

As shown in Fig. 1, compared with the ctrl group, the expression level of MALAT1 was decreased in the CIA group. After PAE treatment, MALAT1 expression was upregulated in CIA rats, and the upregulation amplitude was increased in a PAE concentration-dependent manner. Meanwhile, the apoptosis rate of FLS in CIA rats was observed by TUNEL assay. Compared with the control group, the apoptosis rate was decreased in CIA group, and the number of apoptosis cells increased after PAE intervention(Fig. 2a-b). Apoptosis rate increased after PAE treatment compared with the CIA group. The CIA+PAE-high group significantly promoted the apoptosis rate. The effect of PAE to promote apoptosis of FLS was also elevated in a concentration-dependent manner, which is consistent with our previous study in vitro that PAE upregulates MALAT1.

\section{PAE could allievate arthritis index, degree of paw swelling in CIA rats}

The assessment indicators including arthritis index and degree of paw swelling were used to evaluate the therapeutic effects of PAE. As shown in Fig. 3a, compared with the ctrl group, the ankle joints of CIA rats were significantly swollen, the degree of joint swelling in the CIA group was high throughout the observation period due to successful modeling, nevertheless, the swollen joint could be improved after PAE intervention, with the best improvement in the CIA+PAE-high group (Fig. 3b). The arthritis index of the CIA group was significantly higher from the 10th day, while the CIA rats that had undergone PAE treatment had decreased in all dosage groups, with the highest improvement being observed in the CIA+PAE-high group(Fig. 3c), implying a concentration-dependent improvement in the therapeutic effect of PAE. Fig. 3d revealed the body weights of all rats gradually increased during the experimental observation period, whereas the CIA group lost significantly more weight compared with the ctrl group. 
The degree of weight loss alleviated after PAE intervention, which was related to PAE dose, with the CIA+PAE-high group showing the best improvement in weight loss compared with CIA+PAE-low and CIA+PAE-mid groups.

\section{PAE could ameliorate pathological changes of joints in CIA rats}

As shown in Fig. 4a-b, in the CIA group the tissue structure of the ankle joints showed local moderate destruction of the articular cartilage, which was replaced by fibrous tissue, and the joint synovium was severely hyperplastic, with visible hyperplastic synovium deep into the joint cavity, interstitial severe inflammatory cell infiltration and fibrous tissue proliferation. After PAE treatment, the degree of joint synovial hyperplasia and osteointerstitial inflammatory cell infiltration continuously decreased with the rising drug dose, among which the CIA+PAE-high group displayed the best therapeutic effect, the difference between it and CIA group was statistically significant $(P<0.001)$.

\section{PAE could reduce the expression levels of IL-2, IL-6 and TNF $-a$ in ClA rats}

The expression level of inflammatory cytokine is also an indicator to evaluate the effect of PAE on CIA rats. ELISA was used to detect their expression levels of them. In the CIA group, the levels of IL-2, IL-6 and TNF - a were significantly higher than those in ctrl group $(P<0.01)$ (Fig. 4c-e). Compared with the CIA group, the expression of IL-2, IL-6 and TNF - a decreased significantly after PAE treatment, particularly the CIA+PAE-high group can significantly inhibit these inflammatory factors $(P<0.01)$.

\section{PAE could inhibit the activation of the Wnt $1 / \beta$-catenin pathway}

In order to further explore the mechanism of PAE up-regulating MALAT1 and promoting synovial cell apoptosis, we detected the mRNA and protein expression of wnt, $\beta$-catenin, bax,caspase3,caspase 9 and bcl-2. As shown in Fig. 5a-e, compared with the ctrl group, in the CIA group, the mRNA expression of Wnt1, $\beta$-catenin and bcl-2 increased, while the mRNA expression of bax, caspase 3 and caspase 9 decreased $(P<0.01)$. Compared with the CIA group, after PAE treatment, the mRNA expressions of Wnt, $\beta$ catenin and bcl-2 decreased in a dose-dependent manner, while the mRNA expressions of bax, caspase 3 and caspase 9 increased in a dose-dependent manner $(P<0.05, P<0.01)$.

In Fig. 6, compared with the ctrl group, the protein expression levels of Wnt1, $\beta$-catenin and bcl-2 increased in the CIA group, while the levels of bax, caspase 3 and caspase 9 decreased $(P<0.01)$. Moreover, compared with the CIA group, PAE inhibited protein expression of Wnt1, $\beta$-catenin and bcl-2 in a dose-dependent manner, while the influence of PAE on the protein expression of bax, caspase 3 and caspase 9 increased in a dose-dependent manner $(P<0.05)$.

\section{Discussion}


Studies have demonstrated that FLS highly specialized mesenchymal cells found in the synovium of diarthrodial joints, contribute to the destruction of joint structure in RA[3]. When the joint structure is damaged, the synovial tissue responds as an ill-adapted wound healing response, however if has destructive characteristics and will ultimately lead to irreversible tissue damage to the tendon, cartilage and bone[17]. Therefore, it is important to explore potential therapeutic targets associated with the immune-inflammatory mechanism of the synovial tissue[18]. It has previously been reported that IncRNA MALAT1 is involved in the immune-inflammatory response of RA and is one of the potential therapeutic targets for RA[19]. In this study, we found that the expression level of IncRNA MALAT1 was lower in the CIA group than that in the ctrl group, this was consistent with the downregulation of MALAT1 in peripheral blood mononuclear cells in patients [20].

In recent years, IncRNA MALAT1 has been one of the star molecules in IncRNA research, with most studies mainly focusing on its tumor immune mechanism. It is considered to have significant IncRNAtargeting potential due to the existence of a highly conserved triple helix structure (ENE) at its 3 'terminal $[21,22]$. Therefore, its high conservation is suitable for the study of various immune mechanisms. Studies have been proved that MALAT1 not only regulates apoptosis-related genes by binding to proteins or acting as competitive endogenous RNA[23], but can also be regulated by drugs to affect apoptosisrelated pathways[24]. Herein, results showed that the expression level of MALAT1 was downregulated in RA-FLS in vivo, but its level could be increased by PAE, This finding is consistent with our previous study (currently under review for publication) which found that PAE regulates IncRNA MALAT1 to participate in its treatment of RA in FLS.

In the current study, we explored the therapeutic effect of PAE on CIA rat models. It is worth noting that CIA model has been used extensively to study the mechanism of RA because the model has mature conditions in immunological and pathological features[25]. Our results revealed that PAE reduced the arthritis score. Specifically, histopathology results showed that joint synovial hyperplasia and osteointerstitial inflammatory cell infiltration were reduced after administering PAE, whereas the levels of, the inflammatory cytokines such as TNF - $a$, IL-6 and IL-2, were all decreased by PAE. Collectively, these findings suggest that PAE has a good therapeutic effect on CIA rat models. A previous study revealed that inflammatory cytokines participate in the pathogenesis of joint inflammatory injury in RA, and there is a wide network of cytokines that contribute to mediating cartilage and bone destruction[26]. TNF - $a$ and IL6 are both pro-inflammatory cytokines produced by activated RA-FLS[27]. Besides, IL-2, a cytokine glycoprotein, could stimulate $T$ cells and natural killer cells, and take part in the immune response mediated by the Th1 cytokine pathway [28]. It should be noted that the therapeutic effect of PAE increased in a dose-dependent manner. Although several recent studies that have reported that PAE can ameliorate RA joint inflammation by regulating the function of immune cells or related signaling pathways $[14,29,30]$, the effect of PAE on MALAT1 has rarely been reported. Based on our previous experimental results, we speculated that PAE may regulate the expression level of MALAT1 to treat RA in FLS. This study found that the arthritis score in the CIA group was higher than that in all PAE treatment groups, which suggests that PAE may reduce the production of inflammatory cytokines and improve 
synovial tissue inflammation by upregulating MALAT1 in FLS, thereby decreasing joint arthritis scores in CIA rat models.

Considering the unique aggressive phenotype in RA, cell apoptosis plays an important role in the pathological process of RA-FLS [31]. A previous study proved that inducing the apoptosis of RA-FLS may alleviate the development of RA [32]. The TUNEL staining performed in this study displayed that the cell morphology changed, which indicated cell apoptosis, but the number increased after PAE treatment. Moreover, the apoptosis rate was significantly higher in the CIA group with lower expression of MALAT1 than in all PAE treatment groups, suggesting that PAE could improve the apoptosis of RA-FLS by upregulating MALAT1. Furthermore, we explored the expression levels of $\mathrm{Bcl}-2$, Bax, caspase-3 and caspase-9. As one of the conservative cell death pathways, apoptosis is regulated by Bcl-2(B cell Lymphoma 2) family proteins, which composed of pro-apoptotic (such as Bax and Bak) and prosurvival(such as Bcl-2 and Bcl-XL) members [33]. Notably, when the balance between pro-apoptotic and pro-survival factors is broken, caspase 3 and caspase 9 are activatied and mitochondrial cytochromes are released to promote cracking of the cell structure, which ultimately induces cell apoptosis [34]. Herein, qPCR results indicated that PAE treatment could increase the mRNA expression levels of Bax, caspase 3 and caspase 9, and decrease the mRNA expression level of Bcl-2. Meanwhile, western blot analysis results showed that PAE had the same influence on their protein expression level. Overall, these findings suggest that the mechanism through which PAE alleviates the development of RA may associated with the pathway participating in apoptosis.

Accumulating evidence has suggested that the Wnt/ $\beta$-catenin signaling pathway is activated in the pathogenesis of RA[8,35]. As a conserved evolutionary pathway, it controls a variety of cellular behaviors in RA. Wnt1, a member of the Wnt ligand gene family, abnormally activates the Wnt/ß-catenin pathway and regulates transcription of downstream genes, thereby maintaining cell proliferation, apoptosis, metastasis, and stem cell properties [36-38]. A previous study found that IncRNA MALAT1 affects cell proliferation and apoptosis by inhibiting the Wnt/ $\beta$-catenin pathway, even including reducing cell inflammatory injury [39]. In this study, the mRNA and protein expression levels of Wnt1 and $\beta$-catenin, indicated that the Wnt1/ $\beta$-catenin pathway was significantly activated in the CIA group, but it was inhibited by PAE in a dose-dependent manner. This result suggested that PAE promotes cell apoptosis in CIA rat models by inhibiting activation of the Wnt1/ $\beta$-catenin pathway. Moreover, the expression level of MALAT1 was improved in all PAE treatment groups, whereas the Wnt1/ $\beta$-catenin pathway activity was inhibited in all PAE treatment groups, which further suggests that PAE could enhance inhibition of the Wnt1/ $\beta$-catenin pathway by upregulating MALAT1.

Results obtained in this study demonstrated that PAE had a beneficial therapeutic effect on arthritis in CIA rats. It was evident that PAE could up-regulate the expression level of IncRNA MALAT1 in FLS of CIA rats, reduce the expression levels of inflammatory cytokines such as TNF $-\alpha$, IL-6 and IL-2, and inhibit activity of the Wnt1/ $\beta$-catenin pathway.

\section{Conclusion}


In summary, this study explored the therapeutic effect of PAE on CIA rat models, and found that it could alleviate arthritis in CIA rat models. It was evident that PAE could promote synovial cells apoptosis in CIA and its mechanism of action was through inhibition of the Wnt $1 / \beta$-catenin pathway by upregulating the expression level of IncRNA MALAT1. Collectively, our results suggest that PAE promotes apoptosis of synovial cells and inflammatory infiltration in CIA. This findings provide novel insights for alleviation of joint inflammation by PAE, which suggests that it may be a potential drug targeting IncRNA MALAT1 to treat RA.

\section{Declarations}

Funding: This work was supported by key special disease construction project of the State Administration of Traditional Chinese medicine of the military system (Grant No.2007ZDZB001).

Competing Interests. The authors have no competing interests.

Ethics Approval. The experimental procedures were approved by the Animal Ethics Committee of Jinling Hospital. (NO. 2021DZGKJDWLS-0050).

Animal welfare: The experimental animals' welfare was carried out in accordance with China's National Standard-General Principles for The Welfare of Experimental Animals (No. 20184453-T-469) and the National Institutes of Health Guide for the Care and Use of Laboratory Animals (NIH Publications No.8023, revised 1978) .

Consent to participate. Not applicable.

Consent to publish. Not applicable.

\section{AUTHOR CONTRIBUTIONS}

Fan Yang contributed to design the study, participating in the experiment, analyzing and interpreting data, and drafting the article; Dongyi Wang, Junyi Shen, Jun Han and Kailun Zhou were responsible for the coordination in the experiment and proofreading the data; Zhiming Zhao, Hui Cai and Wei Shang took charge of the conception of the study, supervise the experiment and proofreading data; All the authors above proofread and confirmed the article before submitting.

\section{References}

1. Aletaha, D., and J. S. Smolen. 2018. Diagnosis and Management of Rheumatoid Arthritis: A Review. JAMA 320: 1360-1372.

2. Smolen, J. S., R. B. M. Landewe, J. W. J. Bijlsma, G. R. Burmester, M. Dougados, A. Kerschbaumer, I. B. Mclnnes, A. Sepriano, R. F. van Vollenhoven, M. de Wit, D. Aletaha, M. Aringer, J. Askling, A. Balsa, M. Boers, A. A. den Broeder, M. H. Buch, F. Buttgereit, R. Caporali, M. H. Cardiel, D. De Cock, C. Codreanu, M. Cutolo, C. J. Edwards, Y. van Eijk-Hustings, P. Emery, A. Finckh, L. Gossec, J. E. 
Gottenberg, M. L. Hetland, T. W. J. Huizinga, M. Koloumas, Z. Li, X. Mariette, U. Muller-Ladner, E. F. Mysler, J. A. P. da Silva, G. Poor, J. E. Pope, A. Rubbert-Roth, A. Ruyssen-Witrand, K. G. Saag, A. Strangfeld, T. Takeuchi, M. Voshaar, R. Westhovens, and D. van der Heijde. 2020. EULAR recommendations for the management of rheumatoid arthritis with synthetic and biological diseasemodifying antirheumatic drugs: 2019 update. Ann Rheum Dis 79: 685-699.

3. Nygaard, G., and G. S. Firestein. 2020. Restoring synovial homeostasis in rheumatoid arthritis by targeting fibroblast-like synoviocytes. Nat Rev Rheumatol 16: 316-333.

4. Myasoedova, E., J. M. Davis 3rd, S. J. Achenbach, E. L. Matteson, and C. S. Crowson. 2019. Trends in Prevalence of Functional Disability in Rheumatoid Arthritis Compared With the General Population. Mayo Clin Proc 94: 1035-1039.

5. Pappas, D. A., G. St John, C. J. Etzel, S. Fiore, T. Blachley, T. Kimura, R. Punekar, K. Emeanuru, J. Choi, S. Boklage, and J. M. Kremer. 2021. Comparative effectiveness of first-line tumour necrosis factor inhibitor versus non-tumour necrosis factor inhibitor biologics and targeted synthetic agents in patients with rheumatoid arthritis: results from a large US registry study. Ann Rheum Dis 80: 96-102.

6. Hsieh, P. H., O. Wu, C. Geue, E. McIntosh, I. B. Mclnnes, and S. Siebert. 2020. Economic burden of rheumatoid arthritis: a systematic review of literature in biologic era. Ann Rheum Dis 79: 771-777.

7. Genovese, M. C., K. Kalunian, J. E. Gottenberg, N. Mozaffarian, B. Bartok, F. Matzkies, J. Gao, Y. Guo, C. Tasset, J. S. Sundy, K. de Vlam, D. Walker, and T. Takeuchi. 2019. Effect of Filgotinib vs Placebo on Clinical Response in Patients With Moderate to Severe Rheumatoid Arthritis Refractory to DiseaseModifying Antirheumatic Drug Therapy: The FINCH 2 Randomized Clinical Trial. JAMA 322: 315325.

8. Pan, F., L. Zhu, H. Lv, and C. Pei. 2016. Quercetin promotes the apoptosis of fibroblast-like synoviocytes in rheumatoid arthritis by upregulating IncRNA MALAT1. Int J Mol Med 38: 1507-1514.

9. Su, K., N. Wang, Q. Shao, H. Liu, B. Zhao, and S. Ma. 2021. The role of a ceRNA regulatory network based on IncRNA MALAT1 site in cancer progression. Biomed Pharmacother 137: 111389.

10. Zhang, L., and W. Wei. 2020. Anti-inflammatory and immunoregulatory effects of paeoniflorin and total glucosides of paeony. Pharmacol Ther 207: 107452.

11. Wang, J. S., Y. Huang, S. Zhang, H. J. Yin, L. Zhang, Y. H. Zhang, Y. W. Song, and D. D. Li. 2019. A Protective Role of Paeoniflorin in Fluctuant Hyperglycemia-Induced Vascular Endothelial Injuries through Antioxidative and Anti-Inflammatory Effects and Reduction of PKCbeta1. Oxid Med Cell Longev 2019:5647219.

12. Wen, J., B. Xu, Y. Sun, M. Lian, Y. Li, Y. Lin, D. Chen, Y. Diao, M. Almoiliqy, and L. Wang. 2019. Paeoniflorin protects against intestinal ischemia/reperfusion by activating LKB1/AMPK and promoting autophagy. Pharmacol Res 146: 104308.

13. Han, F., D. Zhou, X. Yin, Z. Sun, J. Han, L. Ye, W. Zhao, Y. Zhang, Z. Wang, and L. Zheng. 2016. Paeoniflorin protects diabetic mice against myocardial ischemic injury via the transient receptor potential vanilloid 1/calcitonin gene-related peptide pathway. Cell Biosci 6: 37. 
14. Zhai, W., Z. Ma, W. Wang, L. Song, and J. Yi. 2018. Paeoniflorin inhibits Rho kinase activation in joint synovial tissues of rats with collagen-induced rheumatoid arthritis. Biomed Pharmacother 106: 255259.

15. Liu, C., Q. Zhao, L. Zhong, Q. Li, R. Li, S. Li, Y. Li, N. Li, J. Su, W. Dhondrup, X. Meng, Y. Zhang, Y. Tu, and X. Wang. 2021. Tibetan medicine Ershiwuwei Lvxue Pill attenuates collagen-induced arthritis via inhibition of JAK2/STAT3 signaling pathway. J Ethnopharmaco/ 270: 113820.

16. Li, H. J., C. T. Zhang, H. Du, T. Xu, Q. Li, P. Wang, G. Fang, and G. Fan. 2019. Chemical Composition of Bawei Longzuan Granule and Its Anti-Arthritic Activity on Collagen-Induced Arthritis in Rats by Inhibiting Inflammatory Responses. Chem Biodivers 16: e1900294.

17. Weyand, C. M., and J. J. Goronzy. 2021. The immunology of rheumatoid arthritis. Nat Immunol 22: 10-18.

18. Chatterjee, S., D. Bhattcharjee, S. Misra, A. Saha, N. P. Bhattacharyya, and A. Ghosh. 2020. Increase in MEG3, MALAT1, NEAT1 significantly predicts the clinical parameters in patients with rheumatoid arthritis. Per Med 17: 445-457.

19. Mishra, S., S. S. Verma, V. Rai, N. Awasthee, S. Chava, K. M. Hui, A. P. Kumar, K. B. Challagundla, G. Sethi, and S. C. Gupta. 2019. Long non-coding RNAs are emerging targets of phytochemicals for cancer and other chronic diseases. Cell Mol Life Sci 76: 1947-1966.

20. Zhang, T. P., B. Q. Zhu, S. S. Tao, Y. G. Fan, X. M. Li, H. F. Pan, and D. Q. Ye. 2019. Long Non-coding RNAs Genes Polymorphisms and Their Expression Levels in Patients With Rheumatoid Arthritis. Front Immunol 10: 2529.

21. Wilusz, J. E., S. M. Freier, and D. L. Spector. 2008. 3' end processing of a long nuclear-retained noncoding RNA yields a tRNA-like cytoplasmic RNA. Cell 135: 919-932.

22. Goyal, B., S. R. M. Yadav, N. Awasthee, S. Gupta, A. B. Kunnumakkara, and S. C. Gupta. 2021. Diagnostic, prognostic, and therapeutic significance of long non-coding RNA MALAT1 in cancer. Biochim Biophys Acta Rev Cancer 1875: 188502.

23. Chen, Y., S. Li, Y. Zhang, M. Wang, X. Li, S. Liu, D. Xu, Y. Bao, P. Jia, N. Wu, Y. Lu, and D. Jia. 2021. The IncRNA Malat1 regulates microvascular function after myocardial infarction in mice via miR-26b5p/Mfn1 axis-mediated mitochondrial dynamics. Redox Biol 41: 101910.

24. Mou, L., L. Liao, Y. Zhang, D. Ming, and J. Jiang. 2021. Ursolic acid ameliorates Nthy-ori 3-1 cells injury induced by IL-1beta through limiting MALAT1/miR-206/PTGS1 ceRNA network and NF-kappaB signaling pathway. Psychopharmacology (Berl) 238: 1141-1156.

25. Piao, X., J. Zhou, and L. Xue. 2021. Triptolide decreases rheumatoid arthritis fibroblast-like synoviocyte proliferation, invasion, inflammation and presents a therapeutic effect in collageninduced arthritis rats via inactivating IncRNA RP11-83J16.1 mediated URI1 and beta-catenin signaling. Int Immunopharmacol 99: 108010.

26. Brennan, F. M., and I. B. McInnes. 2008. Evidence that cytokines play a role in rheumatoid arthritis. $J$ Clin Invest 118: 3537-3545. 
27. Cai, L., Y. R. Mu, M. M. Liu, M. Y. Zhou, B. Meng, F. Y. Liu, and R. Li. 2021. Penta-acetyl Geniposide Suppresses Migration, Invasion, and Inflammation of TNF-alpha-Stimulated Rheumatoid Arthritis Fibroblast-Like Synoviocytes Involving Wnt/beta-Catenin Signaling Pathway. Inflammation 44: 2232-2245.

28. Zhu, H., L. F. Wu, X. B. Mo, X. Lu, H. Tang, X. W. Zhu, W. Xia, Y. F. Guo, M. J. Wang, K. Q. Zeng, J. Wu, Y. H. Qiu, X. Lin, Y. H. Zhang, Y. Z. Liu, N. J. Yi, F. Y. Deng, and S. F. Lei. 2019. Rheumatoid arthritisassociated DNA methylation sites in peripheral blood mononuclear cells. Ann Rheum Dis 78: 36-42.

29. Ma, J., Q. Meng, J. Zhan, H. Wang, W. Fan, Y. Wang, S. Zhang, H. Bian, and F. Zheng. 2021. Paeoniflorin Suppresses Rheumatoid Arthritis Development via Modulating the Circ-FAM120A/miR671-5p/MDM4 Axis. Inflammation 44: 2309-2322.

30. Zhang, L., J. Yu, C. Wang, and W. Wei. 2019. The effects of total glucosides of paeony (TGP) and paeoniflorin (Pae) on inflammatory-immune responses in rheumatoid arthritis (RA). Funct Plant Biol 46: $107-117$.

31. Cai, P., Z. Lu, T. Jiang, Z. Wang, Y. Yang, L. Zheng, and J. Zhao. 2020. Syndecan-4 involves in the pathogenesis of rheumatoid arthritis by regulating the inflammatory response and apoptosis of fibroblast-like synoviocytes. J Cell Physiol 235: 1746-1758.

32. Zhang, Q., J. Liu, M. Zhang, S. Wei, R. Li, Y. Gao, W. Peng, and C. Wu. 2019. Apoptosis Induction of Fibroblast-Like Synoviocytes Is an Important Molecular-Mechanism for Herbal Medicine along with its Active Components in Treating Rheumatoid Arthritis. Biomolecules 9.

33. Singh, R., A. Letai, and K. Sarosiek. 2019. Regulation of apoptosis in health and disease: the balancing act of BCL-2 family proteins. Nat Rev Mol Cell Biol 20: 175-193.

34. Marsden, V. S., L. O'Connor, L. A. O'Reilly, J. Silke, D. Metcalf, P. G. Ekert, D. C. Huang, F. Cecconi, K. Kuida, K. J. Tomaselli, S. Roy, D. W. Nicholson, D. L. Vaux, P. Bouillet, J. M. Adams, and A. Strasser. 2002. Apoptosis initiated by Bcl-2-regulated caspase activation independently of the cytochrome c/Apaf-1/caspase-9 apoptosome. Nature 419: 634-637.

35. Mu, Y. R., M. Y. Zhou, L. Cai, M. M. Liu, and R. Li. 2020. Overexpression of Aquaporin 1 in Synovium Aggravates Rat Collagen-Induced Arthritis Through Regulating beta-Catenin Signaling: An in vivo and in vitro Study. J Inflamm Res 13: 701-712.

36. Sun, J., P. Yan, Y. Chen, Y. Chen, J. Yang, G. Xu, H. Mao, and Y. Qiu. 2015. MicroRNA-26b inhibits cell proliferation and cytokine secretion in human RASF cells via the Wnt/GSK-3beta/beta-catenin pathway. Diagn Pathol 10: 72.

37. Li, B., X. Guo, N. Li, Q. Chen, J. Shen, X. Huang, G. Huang, and F. Wang. 2020. WNT1, a target of miR$34 \mathrm{a}$, promotes cervical squamous cell carcinoma proliferation and invasion by induction of an E-P cadherin switch via the WNT/beta-catenin pathway. Cell Oncol (Dordr) 43: 489-503.

38. Fu, X., L. Hong, Z. Yang, Y. Tu, W. Xin, M. Zha, S. Tu, G. Sun, Y. Li, and W. Xiao. 2020. MicroRNA-148a$3 p$ suppresses epithelial-to-mesenchymal transition and stemness properties via Wnt1-mediated Wnt/beta-catenin pathway in pancreatic cancer. J Cell Mol Med 24: 13020-13035. 
39. Zhang, J., Q. Li, B. Xue, and R. He. 2020. MALAT1 inhibits the Wnt/beta-catenin signaling pathway in colon cancer cells and affects cell proliferation and apoptosis. Bosn J Basic Med Sci 20: 357-364.

Figures

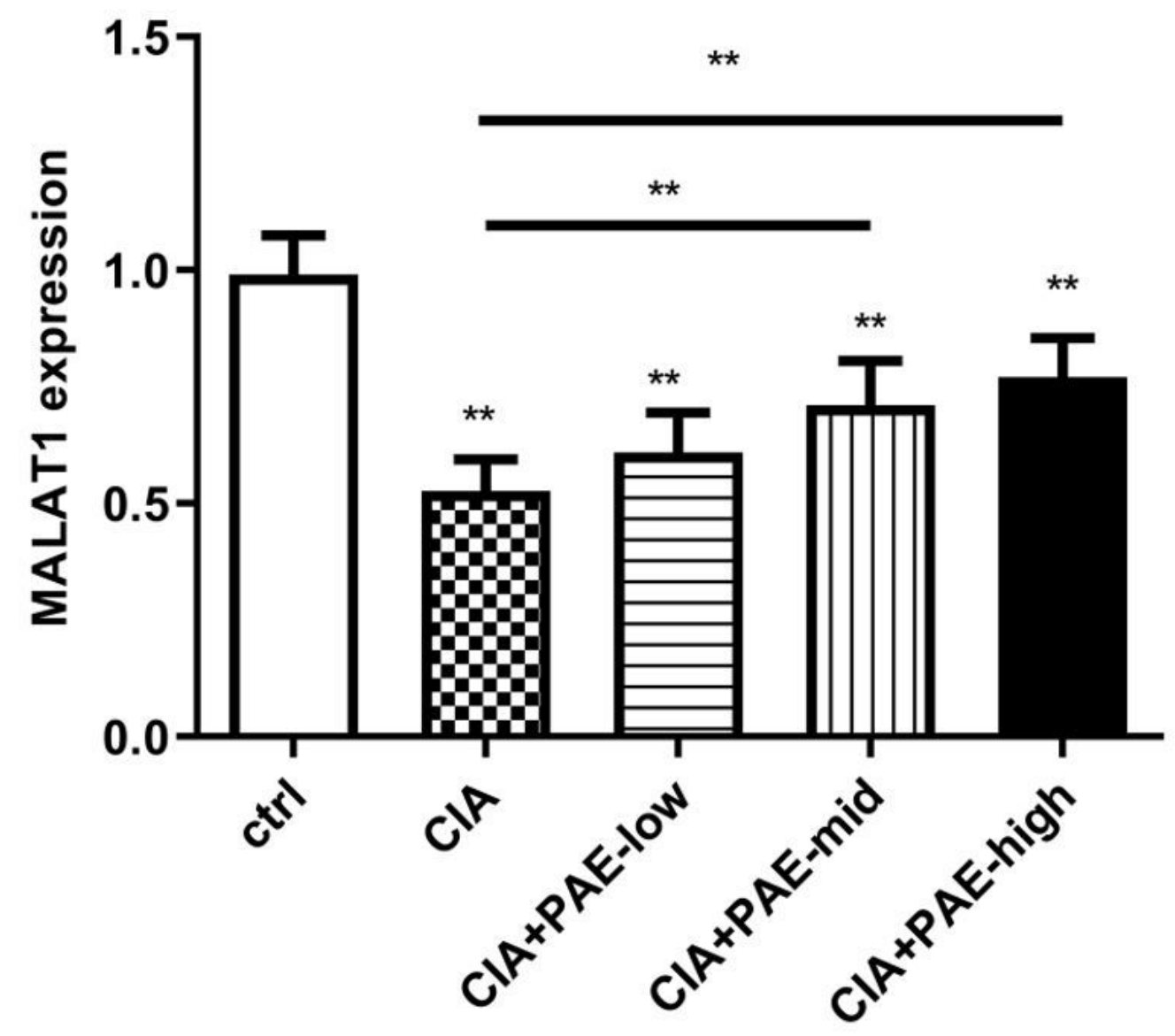

Figure 1

The mRNA expression level of IncRNA MALAT1 in CIA rats. for each group of 3 . ${ }^{*} P<0.05,{ }^{\star} * P<0.01$. 
ctrl

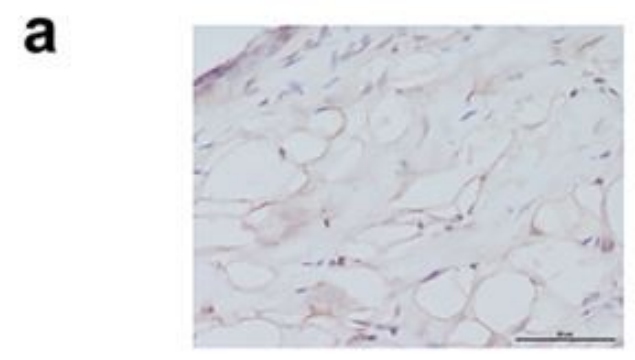

CIA

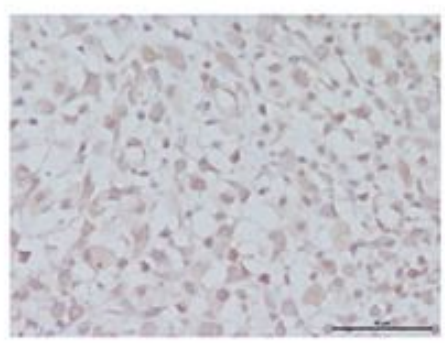

CLA+PAE-mid

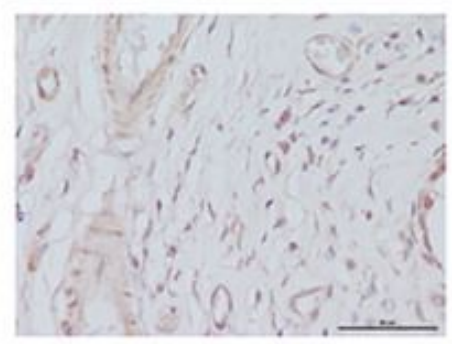

ClA+PAE-high

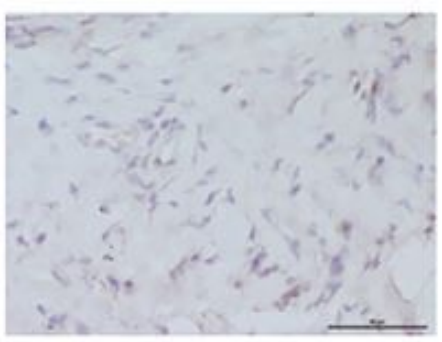

\section{b}

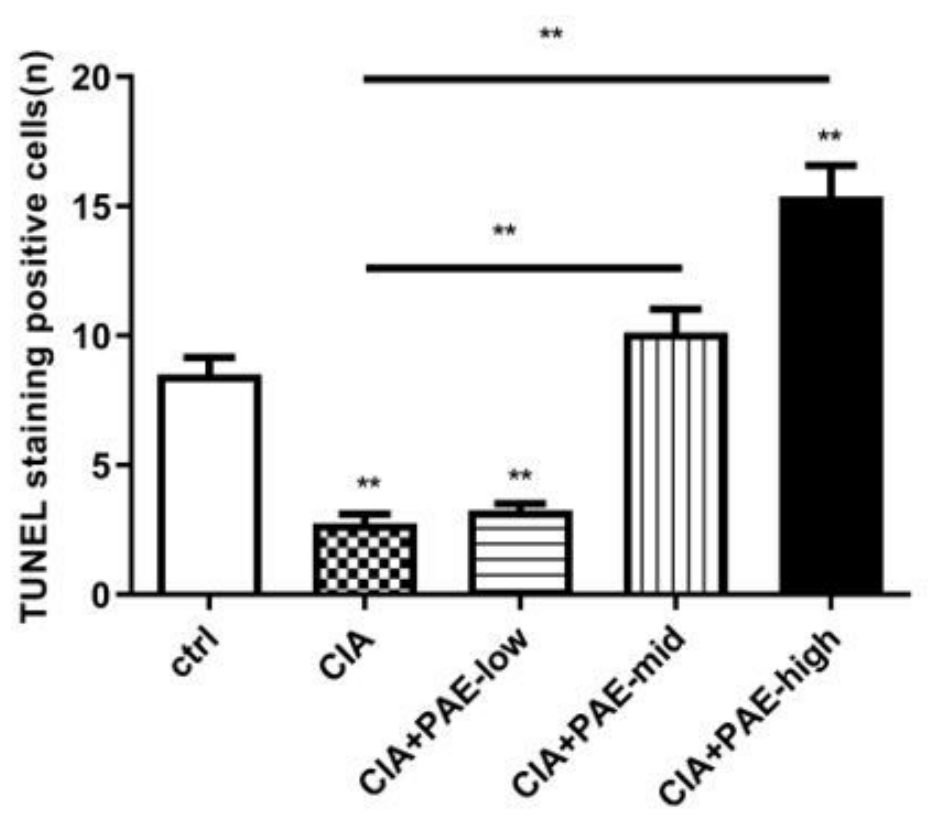

Figure 2

Effect of PAE on the apoptosis rate of FLS in CIA rats. (A) Immunohistochemical pictures of FLS apoptosis by TUNEL $(\times 400)$. (B) Statistic analysis of TUNEL staining positive cells. for each group of 3. * $P<0.05$, ** $P<0.01$. 

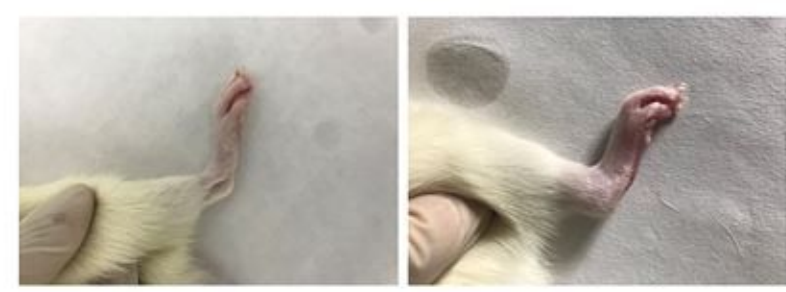

CLA+PAE-IOW

CLA+PAE-mid

CIA+PAE-high
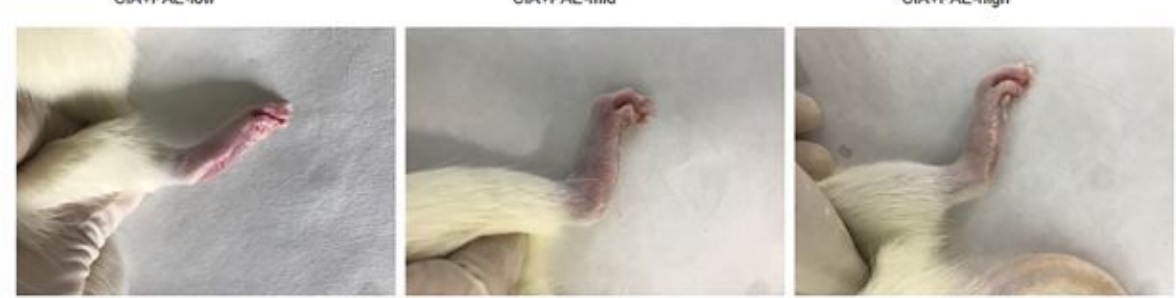

b

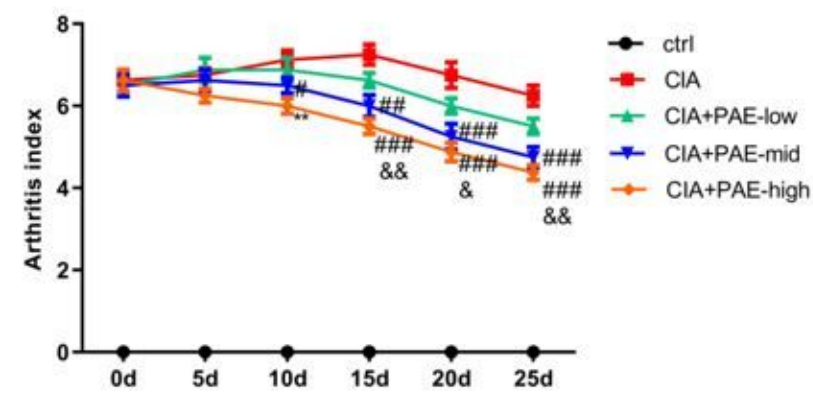

C

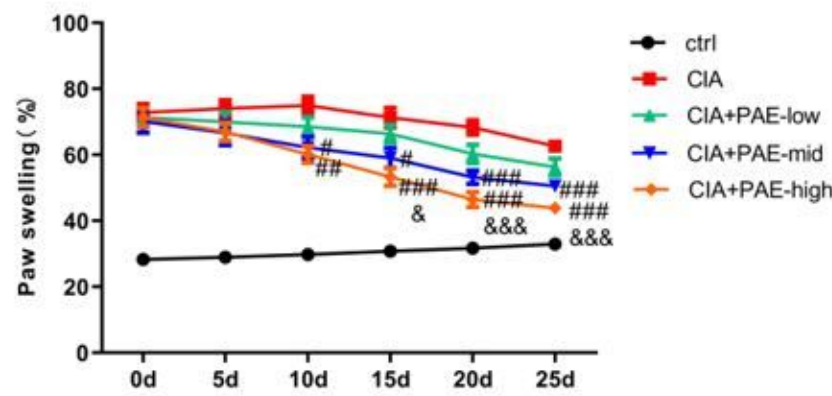

d

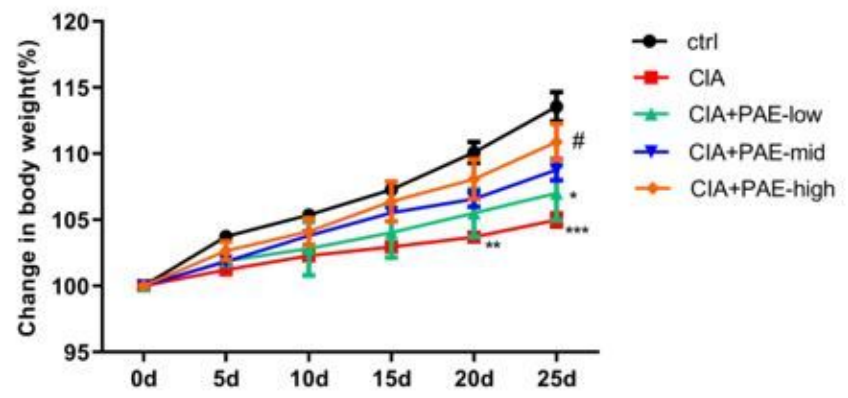

Figure 3

Different treatment on each group. (a) Photos of successful modeling in each treatment group. (b) Arthritis index of each treatment groups. (c) Degree of paw swelling in each treatment groups. (c) Change in body weight of each treatment groups. for each group of 3. Compared with the ctrl group, * $P<0.05$, ** $P<0.01$, ${ }^{\star \star \star} P<0.001$. Compared with the CIA group, \# $P<0.05$, \#\# $P<0.01$,\#\#\# $P<0.001$. Compared with the CIA+PAE-low group, \& $P<0.05, \& \& P<0.01, \& \& \& P<0.001$. 
a
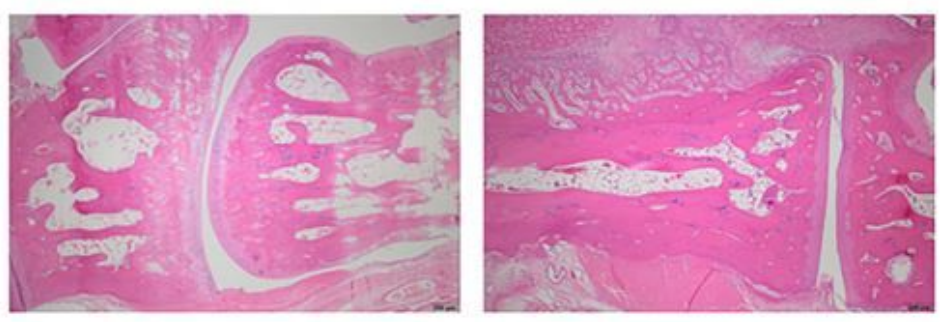

CIA+PAE-low

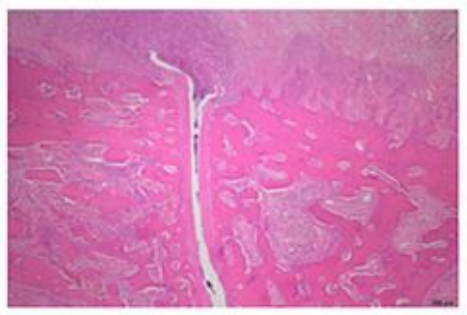

b

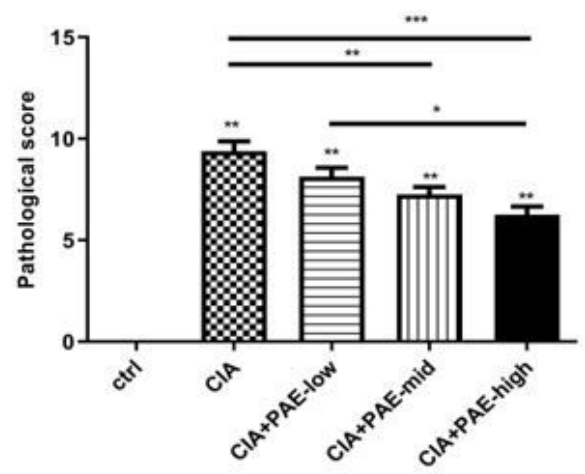

C

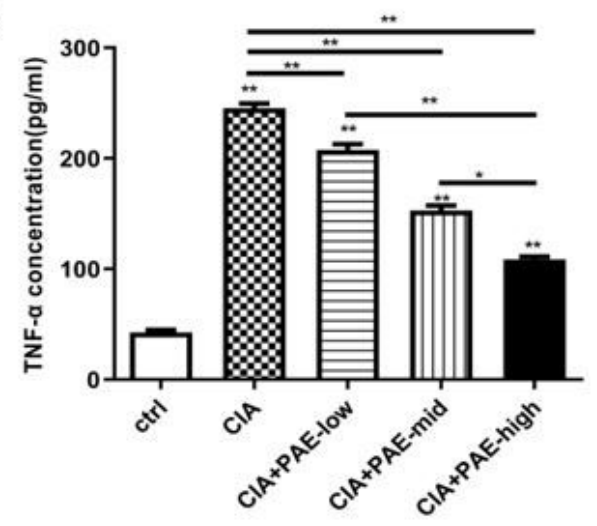

CIA+PAE-mid
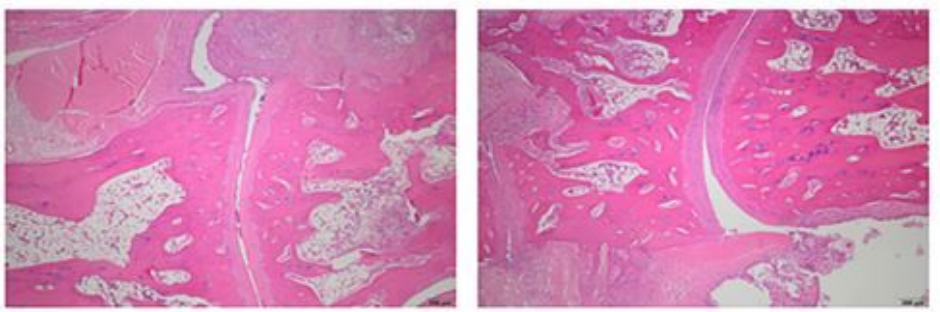

$*$
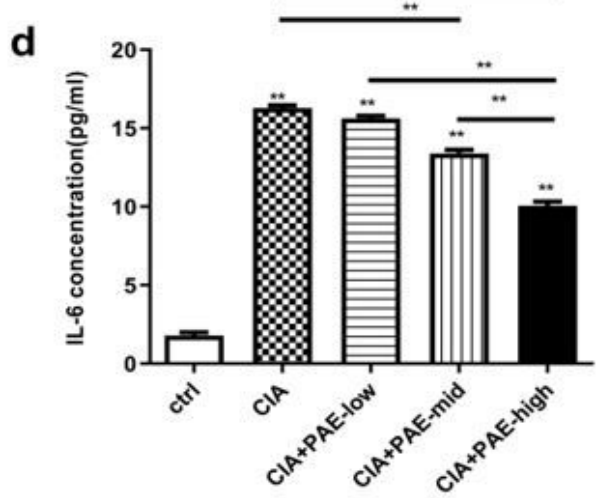

e

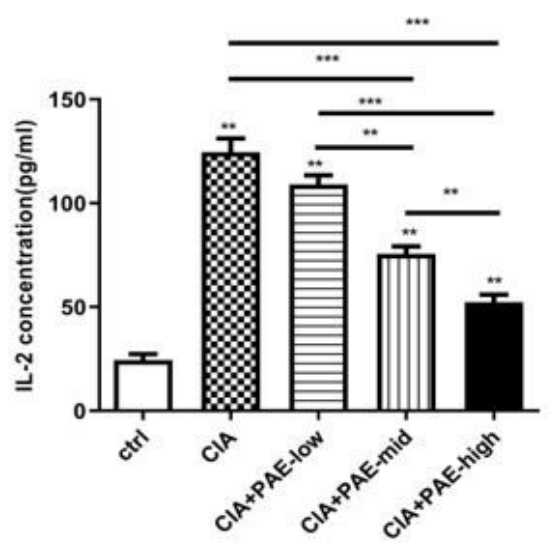

Figure 4

Effects of PAE on changes of inflammation in CIA rats. (a) Representative view under a microscope of HE staining sections of ankle joints $(\times 200)$. (b) Statistics analysis of pathological scores in different groups. (c) Effect of PAE on IL-6. (d) Effect of PAE on TNF -a. (e) Effect of PAE on IL-2. for each group of 3. * $P<0.05$, ** $P<0.01$. 
a

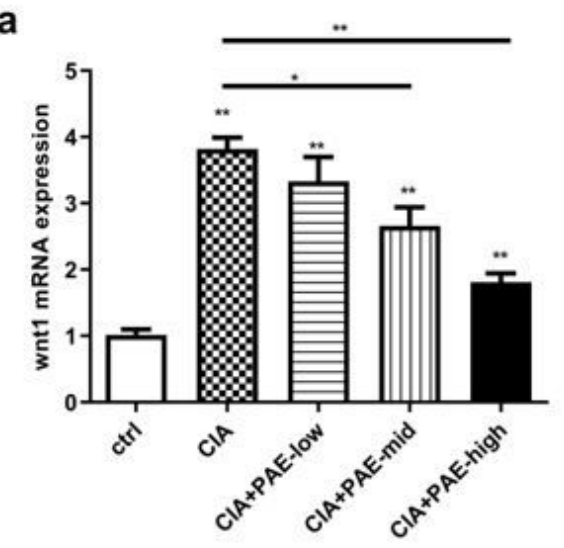

C

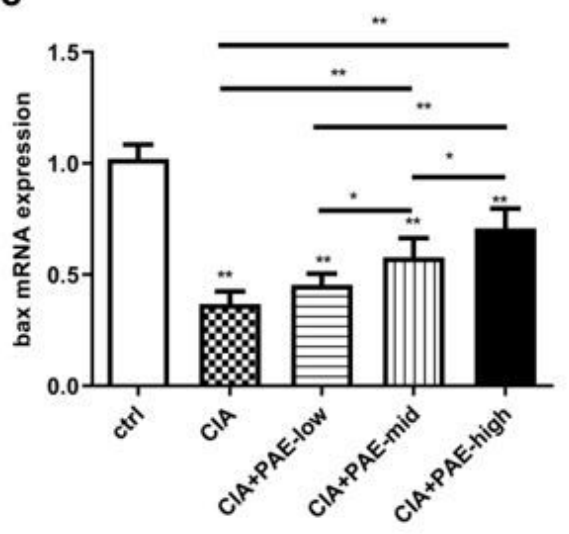

e

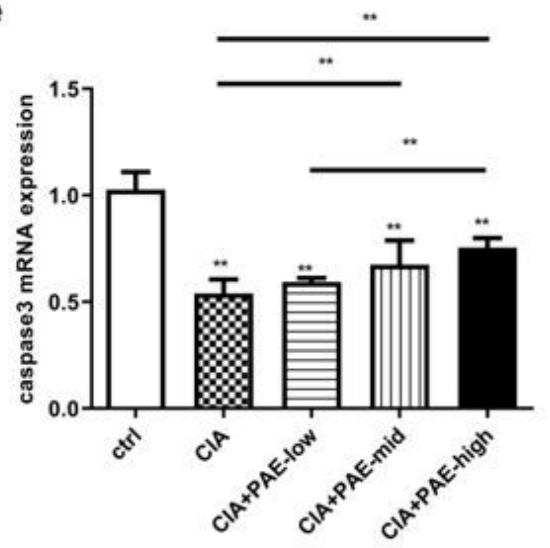

b

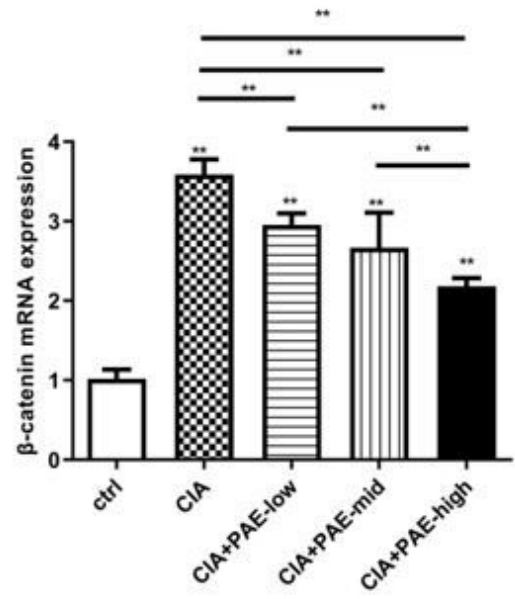

d

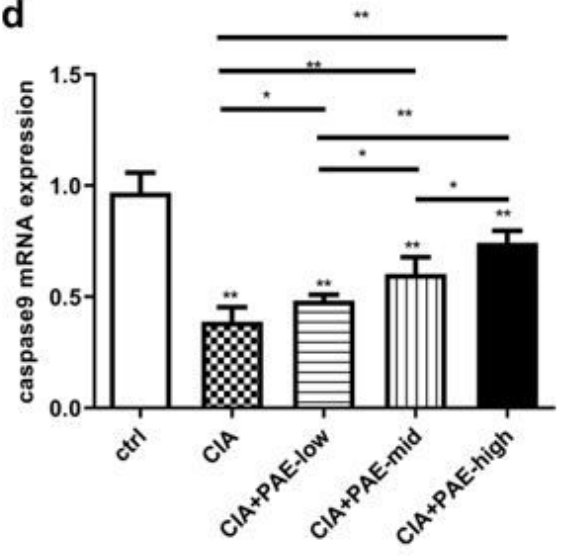

f

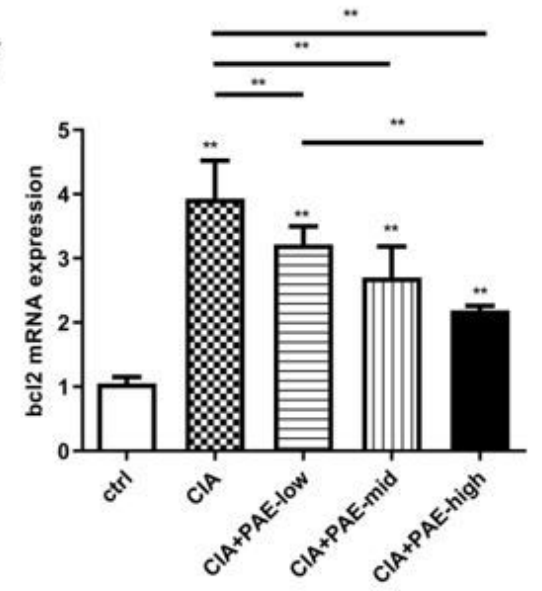

Figure 5

The mRNA expression levels in each group. (a).The mRNA expression of Wnt1. (b) The mRNA exoression of $\beta$-catenin. (c) The mRNA expression of bax. (d) The mRNA expression of caspase 9. (e) The mRNA expression of caspase 3. (f) The mRNA expression of bcl2. for each group of 3 . ${ }^{\star} P<0.05, \star \star ~ P<0.01$. 


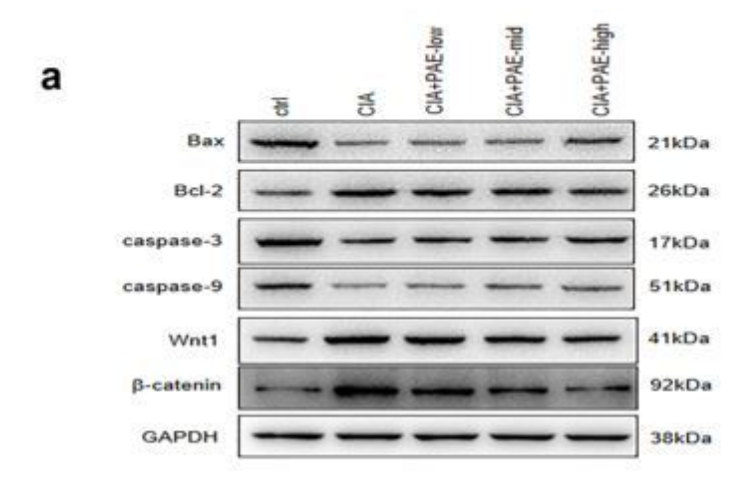

b

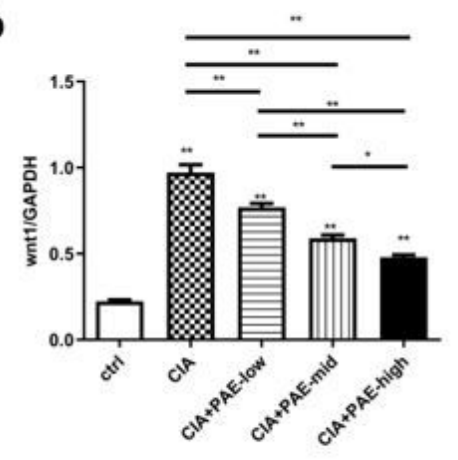

d

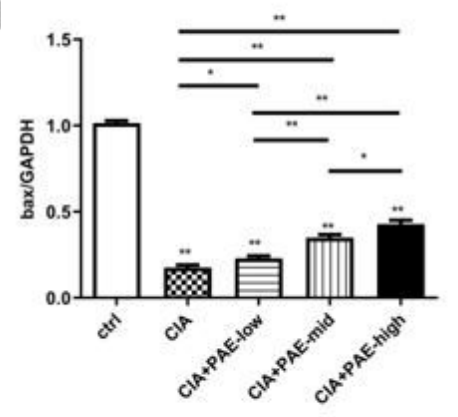

f

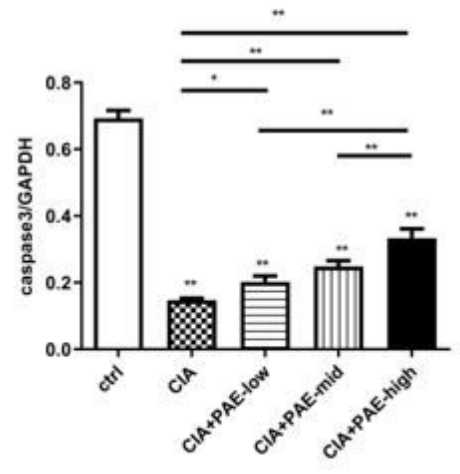

C

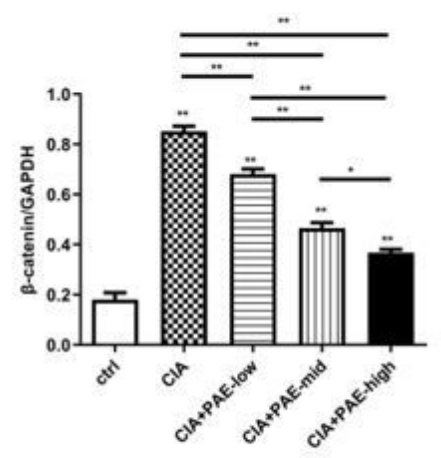

e

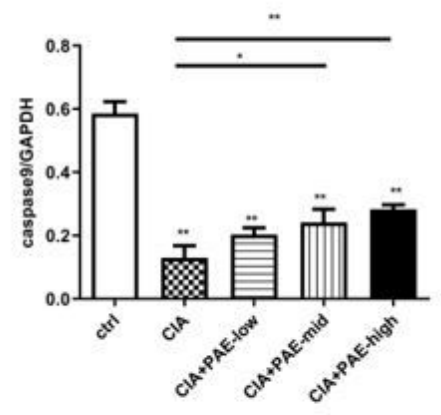

g

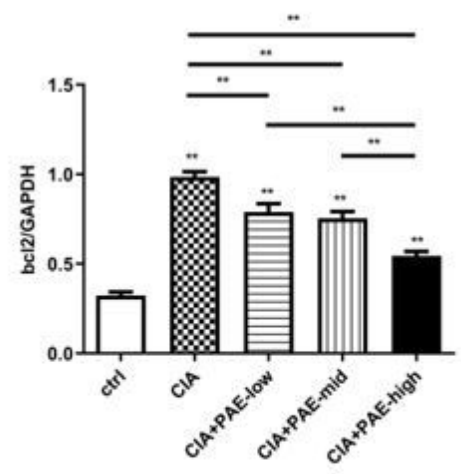

\section{Figure 6}

Western blot analysis of each group. (a) The grey scale of Wnt1, $\beta$-catenin, bax, caspase 9,caspase3, and bcl2. (b) The protein expression of Wnt1. (c) The protein expression of $\beta$-catenin. (d) The protein expression of bax. (e) The protein expression of caspase 9. (f) The protein expression of caspase 3. (g) The protein expression of bcl2. for each group of $3 .{ }^{*} P<0.05, \star \star * 0.01$. 
Page 22/22 